\title{
Development and Research of Electrochemical Cells of Gas Analyzers for Determination of Carbon Oxide in Gas Media
}

\author{
Gulyamov SH.M. ${ }^{1},{ }^{1}$ Tashkent State Technical University, Uzbekistan, gulyamov41@mail.ru \\ Mukhamedkhanov U.T. ${ }^{2}{ }^{2}$ Tashkent State Technical University, Uzbekistan, m-ulugbek@mail.ru \\ Eshmatova B.I. ${ }^{3},{ }^{3}$ Tashkent State Technical University, Uzbekistan, barnoyeshmatova@mail.ru \\ Radjabova M.A ${ }^{4},{ }^{4}$ Tashkent State Technical University, Uzbekistan, maxfuza4717@mail.ru@mail.ru
}

\begin{abstract}
The method for determining the concentration of carbon oxide in vapor-gas mixtures, based on the method of controlled potential amperometry, which belongs to the category of absolute measurement methods, is proposed. Metrological characteristics of electrochemical cells: with diffusion supply of the analyzed gas; with diffusion gas supply; with condensed electrolyte and forced gas supply have been developed and studied.
\end{abstract}

Key words: gas diffusion hydrophobized electrodes, instruments of gas analysis, measuring electrochemical cells.

\section{INTRODUCTION}

Efficiency of operation of the systems for monitoring the state of surrounding air and for monitoring and controlling industrial gas emissions with increasing dataflow is determined by the completeness, reliability and efficiency of obtaining a large number of environmental parameters. The extensive information databases generated in real time are based on data about the content of harmful impurities and components of the air environment. First, this concerns such components as carbon oxide, sulfur compounds, etc., the content of which in industrial emissions is strictly regulated in accordance with the Kyoto Agreement and the instructions of the World Health Organization.

The problem of development of industrial automatic gas analyzers for measuring the content of carbon monoxide in industrial gas emissions and in the air of adjacent industrial zones is of particular urgency. The actuality of problem is confirmed by wide front of scientific research and development aimed at increasing the efficiency of operational monitoring of the state of the surrounding air, reliability and controllability of industrial technological complexes and units based on operational assessment of state of control and operation objects according to the parameters of their functioning, carried out by fast information-measurement control systems complex of operating parameters. [1] The latter include the parameters of content of carbon oxides in composition of industrial gas emissions and exhaust.
In this regard, the efficiency of control and operation of industrial technological installations and complexes with difficult algorithms for processing big operational measuring data, varying loads and performance indicators of monitoring and control objects require the development of effective fast automatic measuring instruments for complex of operating parameters.

Acuteness of the problem is deepened by chronic and widespread shortage of industrial gas analytical instruments for selective measurement of concentration of harmful components in vapor-gas media.

Nowadays, the main directions, which form the basis for the development of devices for monitoring wide class of harmful substances at the level of maximum permissible concentrations (MPC), have been formed. These include, in particular: electrochemical (coulometric, potentiostatic, amperometric) and optical measurement methods.

In theoretical terms, it is vital to study the coulometric method for determining the concentration of the target component in the gas mixture, which ensures high efficiency and selectivity of continuous monitoring of the carbon oxide content in the vapor-gas mixture of industrial process media and in the atmospheric air.

Analysis of patent and technical literature testifies to the broad potentialities of electrochemical cells with gaseous diffused hydrophobized electrodes (GHE), which have not been fully exhausted. The task is that it is necessary to modernize the previously developed primary measuring transducers in such a way that to significantly expand the range of detection of microconcentrations of harmful gases with the smallest overall dimensions of the sensor [2].

All this requires the creation of new generations of gas analyzers for modern information and control systems based on modern physical-chemical measurement methods using the latest advances in science and technology.

We have studied a potentiometric method for determining the concentration of carbon dioxide in the composition of a gas mixture in order to develop on these basis automatic industrial gas-analytical devices.

Statement of the research problem. 
Nowadays, many types of instruments and installations with various analytical capabilities have been developed and used for the analysis of gaseous technological media. The choice of the type of equipment that is optimal for each specific case depends on the nature of the measured object, determined components, requirements for sensitivity, accuracy, speed, cost of analysis, etc. [3].

Determination of carbon oxide content in gas mixtures has a special role in modern industrial production and environmental monitoring systems, where it is important to be able to determine them with high accuracy, reliability and in the shortest possible time.

The general trend towards the maximum automation of the analysis is also traced in most gas analyzers for the determination of $\mathrm{CO}$ microconcentrations in technological gas media. Most of these analyzers are equipped with microprocessors. However, in various instruments, the microprocessor performs a variety of functions - from the broadest and most complete to performing limited functions (such as automatic temperature compensation, automatic linearization of the analyzer sensor and calibration, fixing the sample number, date, month of analysis, etc.). The functions of the microprocessor include continuous monitoring and correction of all factors that may affect the correctness and reproducibility of the analysis (for example, ambient temperature, and gas flow pressure). Any deviation from the norm causes a corresponding correction in the calibration of the analyzer and, therefore, does not affect the accuracy of the analysis. It is also desirable to have a special troubleshooting program that contains ready-made possible solutions, so that the source of the malfunction can be easily found and eliminated. All this makes the developed device reliable in operation [4], easy to maintain and allows achieving high accuracy in determining the measured component in the gas mixture.

Analysis of the current state of gas analytical technology and trends in its further development, as well as the metrological capabilities of linear colorimetric, thermochemical, photocolorimetric, chromatographic, optoacoustic and electrochemical methods of gas analysis indicates significant, far from fully used potential capabilities of electrochemical and optical methods of gas analysis [5].

One of the most perspective ways the development of instruments for measuring the concentration of $\mathrm{CO}$ in gas mixtures based on electrochemical methods of gas analysis and providing the necessary measurement sensitivity, selectivity of the measuring process due to the targeted selection of the catalyst and reasonable development of the design of the sensitive element of the electrochemical cell.

The electrochemical method of gas analysis chosen for research allows developing portable small-sized gas analyzers for determining the microconcentrations of carbon oxide, designed to protect the personnel of many enterprises in various industries from the danger of poisoning, which fully meets the tasks of protecting the health and life of workers.

\section{STUDY OF ELECTROCHEMICAL CELLS OF GAS ANALYSIS DEVICES}

Gas-diffused hydrophobized electrodes (GHE) are widely used in analytical instrument engineering for the determination of microconcentrations of harmful substances in air and technological media, including the determination of the microconcentration of carbon oxide [6].

To analyze the content of carbon monoxide, we have used GHE based on highly dispersed platinum powder, and fluoroplastic as a water repellant (Fig. 1). The electrodes were made by applying a hydrophobized catalyst on a porous fluoroplastic (1) base, which was a homogeneous mixture of highly dispersed platinum powder mixed with a fluoroplastic (2) F-4D suspension in a certain ratio. After drying, the electrodes were sintered at a temperature of $(360 \pm 5){ }^{\circ} \mathrm{C}$ for 15 min. A porous fluoroplastic base serving as a diffusion barrier for gas and at the same time as a liquid seal layer was manufactured by pressing under a pressure of $80 \div 250 \mathrm{kgf} / \mathrm{cm} 2$ from granular fluoroplastic with a fraction of $0.3 \div 0.5 \mathrm{~mm}$.

a)
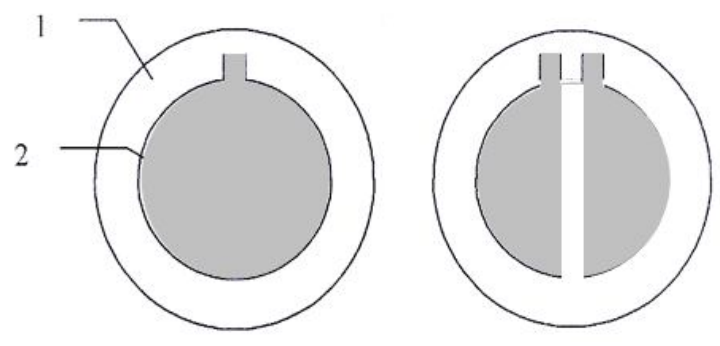

b)

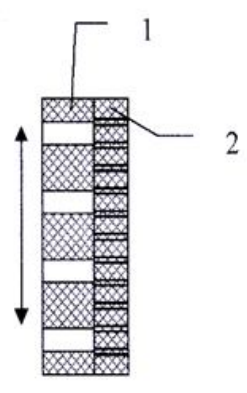

Figure 1: Gas-diffused hydrophobized electrode: auxiliary electrode a) main electrode

b) reference electrode and

Previously developed electrochemical cell (ECC) with diffusion gas supply is shown in Fig. 2. It has a built-up structure made of organic glass and consists of the following main parts: body 1, cover 2, bushings 3 and electrodes 4 and 5 . The electrodes are sealed with rubber rings. The down conductor is carried out by pressing a section of the active surface (active mass) of the electrode to the cell. Filling with an electrolyte solution of $27 \%$ sulfuric acid is carried out through the hole closed with plug 10.

The cell works as follows [7]. The analyzed gas is supplied along the surface of the porous fluoroplastic base of the 
working electrode, and then diffuses through the pores to the hydrophobized catalyst, and the carbon monoxide contained in it is oxidized to dioxide by the reaction:

$$
\mathrm{CO}+\mathrm{H}_{2} \mathrm{O} \rightarrow \mathrm{CO}_{2}+2 \mathrm{H}^{+}+2 \bar{e} .
$$

The cathodic reaction of oxygen reduction in the air with the formation of water occurs on the auxiliary electrode of the cell:

$$
\frac{1}{2} \mathrm{O}_{2}+2 \mathrm{H}^{+}+2 e \rightarrow \mathrm{H}_{2} \mathrm{O} \text {. }
$$

If no other reactions are taking place in the system, then the current passing through the cell is a measure of the concentration of carbon oxide in the sample gas.

During the experiments with ECC, the working electrode potential was set relative to the reference electrode using a P5848 potentiostat in combination with KSP2-003 electronic recorder. The reference electrode and at the same time the auxiliary electrode served as an electrode, which is similar in design to the working electrode with the only difference that the hydrophobized catalyst is divided into two non-contacting parts, one of which served as a reference electrode, and the other served as an auxiliary electrode [8].

To determine the optimal value of the working electrode potential, polarization curves were taken in the range $0 \div 0.1 \mathrm{~V}$ (Fig. 3). The polarization potential was changed stepwise every $0.005 \mathrm{~V}$ with holding at each potential value for 4 hours. (the time required to establish a steady state of the electrode). During the recording of the polarization curves, nitrogen was continuously passed through the chamber of the working electrode, and then for 15 min a gas mixture $\mathrm{N} 2+\mathrm{CO}$ with a carbon monoxide concentration of $51 \mathrm{mg} / \mathrm{m} 3$ was passed.

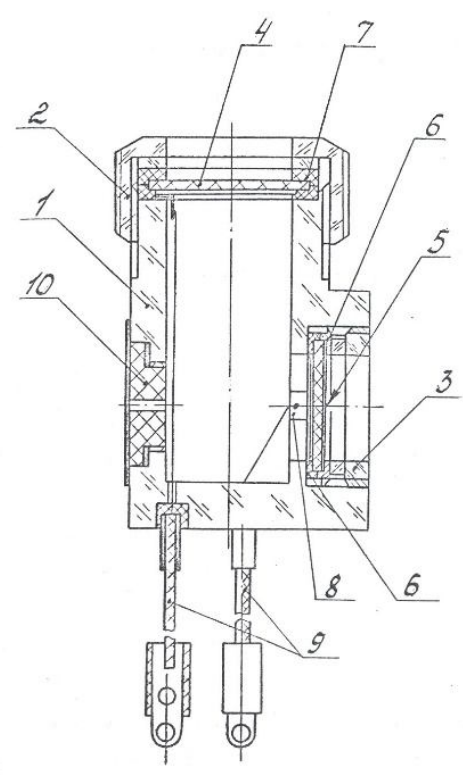

Figure 2: Electrochemical cell with diffusion supply of gas
1 - case; 2 - cover; 3 - bushing;

4, 5 - electrodes; 6,7 - rubber rings;

8 - platinum foil; 9 - wires; 10-plug

$$
\text { I, } \mu \mathrm{A} / \mathrm{cm}^{2}
$$

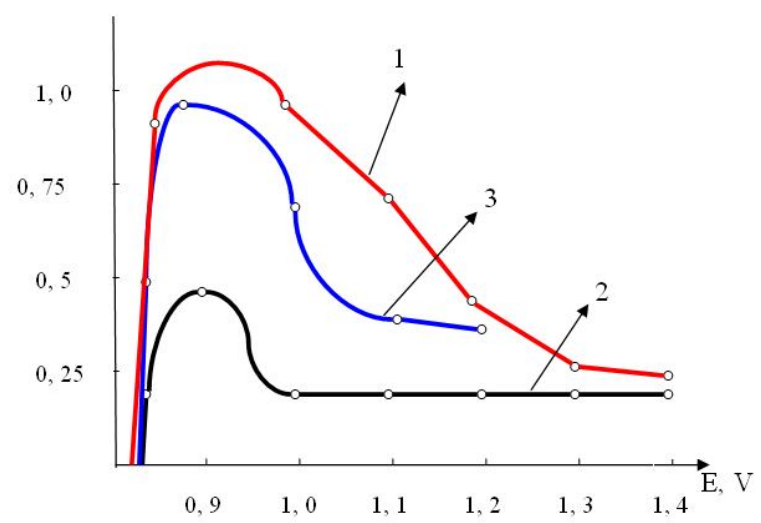

Figure 3: Polarization curves GHE based on Pt $20 \%$ in $\mathrm{H}_{2} \mathrm{SO}_{4}$

\section{DISCUSSION OF RESEARCH RESULTS}

Based on experimental data, we have proposed a hydrophobized platinum electrode as a reference electrode in contact with atmospheric oxygen, which retains a stationary value of the potential for more than two years. The temperature dependences of the rate of carbon oxide oxidation on gasdiffused hydrophobized electrodes are studied. Changes in the value of the $\mathrm{CO}$ oxidation current at different temperatures and a constant value of the potential of the working electrode relative to $\mathrm{E}$ is also equal to the $\mathrm{CO}$ concentration, it is possible, based on experimental data, to determine the activation energy $\mathrm{A}$, which is a function of temperature. To determine the activation energy, we took the temperature dependence of the output signal. The polarization potential was fed to the working electrode from a P-5848 potentiostat.

The output signal values were recorded on the tape of KSP-4 recording potentiometer. The temperature of the cells was changed using a thermostat with holding at each temperature value for $3 \mathrm{~h}$ to establish a stable thermal equilibrium. When determining the temperature dependence, a calibration gas mixture with a carbon monoxide concentration of $22 \mathrm{mg} / \mathrm{m} 3$ was used.

It was determined that the activation energy for different cells is different and is in the range of $0.99 \div 2.0 \mathrm{kcal} / \mathrm{mol}$. These values of the activation energy correspond to the course of the mass transfer process as a whole in the diffusion region (the limiting stage is diffusion, since in the kinetic mode the generation of the activation energy current is higher than 10 $\mathrm{kcal} / \mathrm{mol}$ ).

The influence of the process of transfer of the reactant from the gas flow through the porous hydrophobic base GHE, i.e. influence of the external diffusion stage of the process on the sensor output signal. The theoretical value of the limiting 
diffusion current through a porous membrane can be calculated according to Fick's law [9]:

$$
I_{\lim }=n \cdot F \cdot \tilde{D} \cdot \frac{C_{0}}{\delta}
$$

where $n$ - the number of electrons participating in the reaction of one CO molecule; $F$ - Faraday constant, A s/mol; $\tilde{D}-D q / \beta$ - effective diffusion coefficient, $\mathrm{cm}^{2} / \mathrm{s} ; D-$ diffusion coefficient, $\mathrm{cm}^{2} / \mathrm{s} ; q$ - porosity coefficient; $\beta$ convolution coefficient; $c_{0}-$ concentration, $\mathrm{mol} / \mathrm{cm}^{3} ; \delta$ thickness of hydrophobic base, $\mathrm{cm}$.

Numerical estimate of the limiting diffusion current according to equation (3) at $n=2: \mathrm{D}=0.2 \mathrm{~cm} 2 / \mathrm{s} ; \mathrm{q}=0.3 ; \beta=3 ; \mathrm{F}=1 \times 10-5$ $\mathrm{As} / \mathrm{mol} ; \mathrm{co}=22 \mathrm{~mol} / \mathrm{cm} 3 ; \delta=5.5 \times 10-2 \mathrm{~cm}$ results $\mathrm{Jlim}=2.98$ $\times 10-6 \mathrm{~A} / \mathrm{cm} 2$. For electrodes at the same concentration, the current density is actually obtained $I_{\text {lim }}=1,2 \div 1,6 \times 10^{-6} \mathrm{~A} / \mathrm{cm}^{2}$.

Thus, the experimental value of the real current density is comparable with the theoretical one, and this indicates that the external diffusion stage of the transfer of the reactant through the hydrophobic layer of the electrode is not limiting.

It was established that the optimal porosity of the active mass of the electrode is achieved already at a concentration of 5\% of the hydrophobizing agent, and decrease in the output signal and an increase in the ohmic resistance of the active layer are observed at a concentration of $15 \%$ of the hydrophobizing agent. It can be assumed that it is precisely the increase in the ohmic resistance of the active layer that brings the process of $\mathrm{CO}$ electrochemical oxidation to kinetic limitations.

In order to identify the optimal GHE design, we made 50 electrodes, on which we studied the effect on the limiting oxidation current of the thickness of the gas-permeable base, the pressing pressure, the amount of catalyst per unit of visible surface (Fig. 4 and Fig. 5). As expected, the limiting $\mathrm{CO}$ oxidation current decreased with increasing thickness of the gas-permeable base. Fig. 4 shows that the maximum value of the limiting current of $\mathrm{CO}$ oxidation was observed at a base thickness of $0.25 \mathrm{~mm}$, but the mechanical strength of the base was low and the porosity was uneven. A through flow of electrolyte was observed in all three electrodes after a few days. In addition, during pressing, difficulties arose associated with ensuring uniform distribution of the fluoroplastic powder in the mold. For these reasons, for further studies, the thickness of the gas-permeable base was $0.5 \mathrm{~mm}$. These electrodes have a lower limiting $\mathrm{CO}$ oxidation current, but there are no negative effects typical for bases with a thickness of $0.25 \mathrm{~mm}$.

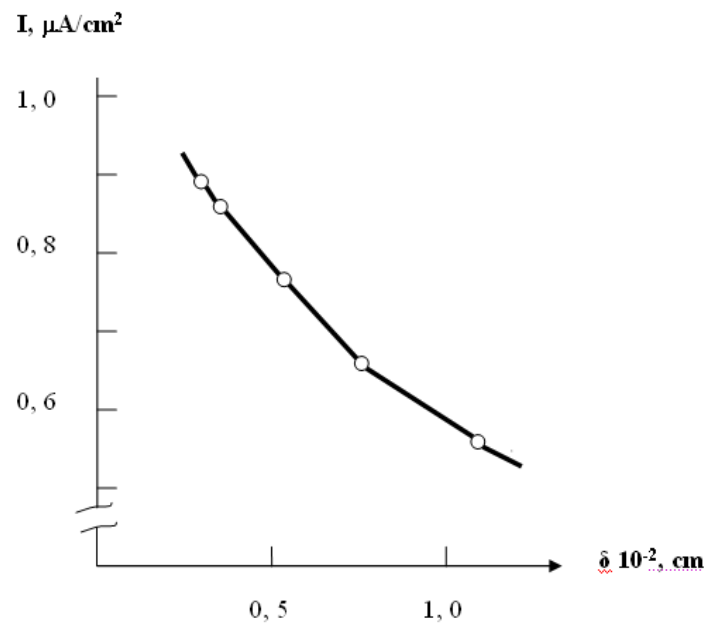

Figure 4: Dependence of the limiting CO oxidation current

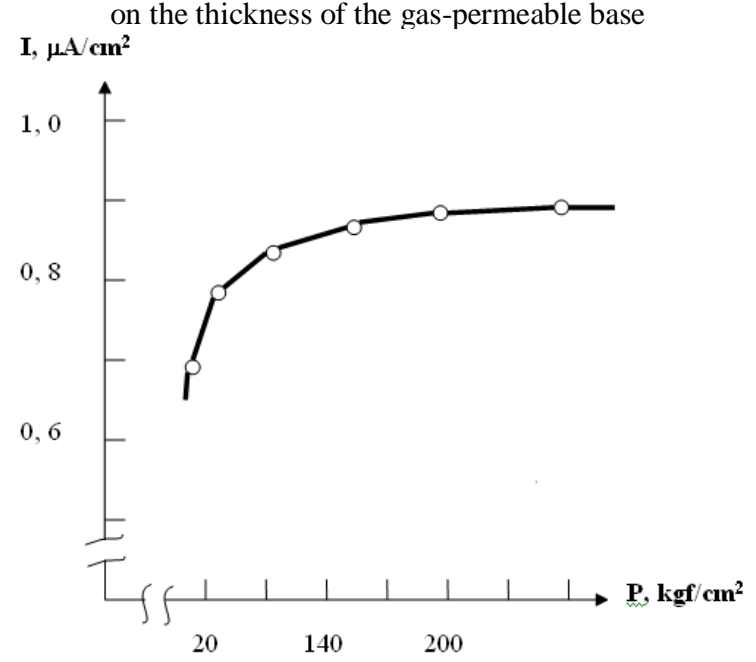

Figure 5: Dependence of the limiting CO oxidation current on the pressing pressure of the gas-permeable base

As can be seen from Fig. 5, the maximum value of the limiting current of $\mathrm{CO}$ oxidation was observed at a compaction pressure of $250 \mathrm{kgf} / \mathrm{cm}^{2}$. It should be noted that the pressing pressure affects both the thickness of the gas-permeable base and its porosity. With an increase in the pressing pressure, the porosity and thickness of the base decrease [10-11]. At low pressing pressures, the base was loose and fragile.

The dependence of the $\mathrm{CO}$ oxidation current on the amount of catalyst applied per unit electrode surface is very weak, as follows from Fig. 6. However, on electrodes with a large amount of catalyst, a uniform distribution of the active mass on the surface and the lowest ohmic resistance are achieved. 


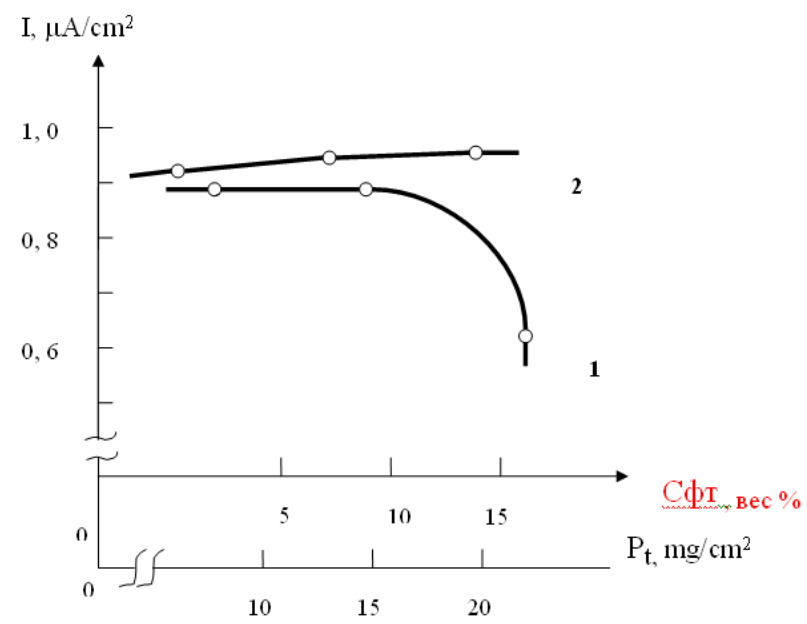

Figure 6: Dependence of the limiting CO oxidation current 1 - on the concentration of the water repellent; 2 - on the amount of platinum per unit of electrode surface

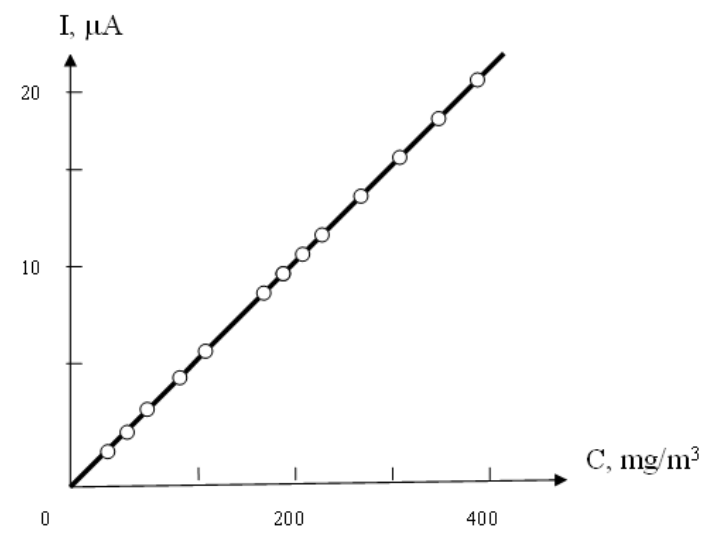

Figure 7: Dependence of the cell output signal on the CO concentration

Based on experimental data [12-13], the following rational constructive and technological parameters of the sensitive element were determined: thickness of the gas-permeable base $5.5 \times 10^{-2} \mathrm{~cm}$; pressing pressure $250 \mathrm{kgf} / \mathrm{cm}^{2}$; the concentration of the hydrophobizing agent in the active mass is $5.0 \%$, the amount of catalyst per $1 \mathrm{~cm}^{2}$ of the electrode surface is 20 $\mathrm{mg} / \mathrm{cm}^{2}$ for freshly prepared electrodes with the specified parameters and the specific surface of the platinum catalyst $16 \div 18 \mathrm{mg} / \mathrm{cm}^{2}$ Pt. The actually obtained current density at a carbon monoxide concentration of $36 \times 10^{-11} \mathrm{~mol} / \mathrm{cm}^{3}(1,0$ $\mathrm{mg} / \mathrm{cm}^{3}$ ) was $1.5 \div 1.6 \mu \mathrm{A} / \mathrm{cm}^{2}$, which quite satisfies the requirements for the value of the conversion coefficient when measuring the $\mathrm{CO}$ concentration in the range of $1 \div 400 \mathrm{mg} / \mathrm{m} 3$.

The dependence of the ECC output signal on the CO concentration (Fig. 7) was determined using the optimal design of the sensitive electrode. As can be seen from the figure, the output signal in the studied concentration range is proportional to the carbon oxide content. [14] The variation of the output signal was no more than $1.0 \%$ of the measured value of the carbon monoxide concentration, and the measurement error, calculated by the methods of mathematical statistics, in the range $12,5 \div 40 \mathrm{mg} / \mathrm{m}^{3} \mathrm{CO}$ did not exceed $\pm 2.0 \%$ of the upper measurement limit. In the concentration range $40 \div 400 \mathrm{mg} / \mathrm{m}^{3}$ $\mathrm{CO}$, the measurement error did not exceed $\pm 0.5 \%$.

\section{CONCLUSION}

The main results of the work are as follows:

1. The characteristics and the possibilities of thermochemical, photocolorimetric, linear-colorimetric, chromatographic, optical-acoustic and electrochemical methods for measuring the concentration of carbon monoxide in technological gas mixtures have been analyzed and studied. 2. The method for determining the concentration of carbon oxide in vapor-gas mixtures, based on the method of potentiostatic amperometry, which belongs to the category of an absolute measurement method and allows one to provide the required metrological characteristics of gas analyzers have been proposed.

3. Research to identify the optimal operating modes of gas-diffused hydrophobized electrodes has been carried out and their rational design has been selected.

4. Experimental methods to select the qualitative and quantitative composition of the catalyst for the sensitive electrode of the electrochemical cell, providing the required measurement selectivity, have been implemented.

5. Metrological characteristics of electrochemical cells based on gas-diffused hydrophobized electrodes have been studied, and the possibilities of implementing optimized variants of their design have been determined.

6. Metrological characteristics of four designs of electrochemical cells (ECC with diffusion supply of the analyzed gas; universal ECC with diffusion gas supply; smallsized ECC with condensed electrolyte and ECC with forced gas supply), allowing to significantly increase the service life of gas analysis devices, have been developed and studied.

\section{REFERENCES}

1. Yusupbekov N.R., Gulyamov Sh.M., Bandenok E.Yu., Yeshmatova B.I. Requirements for electrochemical cells that implement the coulometric titration method // Actual problems in the humanities and socialeconomic sciences: Collected papers. Tashkent, 1999, p.102-106.

2. Gulyamov Sh.M., Yeshmatova B.I., Mamadjanov Kh.A., Mukhamedkhanov U.T., Bandenok E.Yu. Plant for systems of metrological support of gas analysis // International scientific journal "Control systems and machines". Kiev, 2000, No.3, p. 70-72.

3. Yusupbekov N.R., Mukhamedkhanov U.T., Yeshmatova B.I., Matyakubova P.M. Interaction of means of intellectual support in systems of automated control and operation // Mathematical methods in engineering and technology MMTT-2000: Collection of papers of International scientific conference. Saint-Petersburg, 2000. Vol. 6, p. 189-191. 
4. Bandenok E.Yu., Yeshmatova B.I., Dobrev E.N. Electrochemical cell for determining the microconcentration of carbon oxide // Actual problems in the field of technical and fundamental sciences: Collection of papers. Tashkent, 2001, p. 62-64.

5. Bandenok E.Yu., Yeshmatova B.I., Afonin D.V., Yakupova Kh.S. Devices for determining the components of gaseous media of technological processes // "Uzbek journal of oil and gas". Tashkent, 2002. No.2, p. 44-45.

6. Bandenok E.Yu., Afonin D.V., Yeshmatova B.I., Yakupova Kh.S. Multicomponent gas analyzer for monitoring carbon oxide, carbon dioxide, sulfurous gases, hydrocarbons and oxygen // "Uzbek chemical journal". Tashkent, 2002. No. 5, p. 82-85.

7. Mukhamedkhanov U.T., Kimizbaeva A.E., Matyakubova P.M., Yeshmatova B.I. Some principles of building layered information-measuring systems of ensuring the quality industrial products // Infocommunication and computing technologies in science, engineering and education: Reports and abstracts of international scientific conference. September 28-30, 2004. Tashkent, 2004, p. 136-137.

8. Yusupbekov N.R., Gulyamov Sh.M., Bandenok E.Yu., Mukhamedkhanov U.T., Yeshmatova B.I. Industrial gas analyzers of the "MAG" series // International scientific journal "Industrial ACS and controllers". Moscow, 2006. - No. 2. - P.47-49.

9. Mukhamedkhanov U.T., Yeshmatova B.I., Bandenok E.Yu. Development of system for metrological support of gas analysis // Scientific and technical journal "Chemical technology. Control and operation". Tashkent, 2006. No. 1, p. 41-44.

10. Yusupbekov N.R., Gulyamov Sh.M., Mukhamedkhanov U.T., Yeshmatova B.I. Instrumental implementation of coulometric gas analysis // Monthly scientific, technical, production and reference magazine "Pribory" No. 3 Moscow -2020 pp.17-21

11. Yusupbekov N.R., Mukhamedkhanov U.T., Yeshmatova B.I. Research of functioning of electrochemical measuring cells in composition of gas analyzers // Perspective development of science, technical equipment and technology. Collection of scientific papers of the 9th international scientific and practical conference. November 1, 2019. Kursk-2019, p. 363 - 369

12. Yusupbekov N., Igamberdiev H., Mamirov U. Algorithms of sustainable estimation of unknown input signals in control systems // Journal of MultipleValued Logic and Soft Computing, 2019, 33(1-2), p. 110

13. Yusupbekov N., Gulyamov S., Kasymov S., Usmanova N., Mirzaev D. Software implementation of exchange processes in a distributed network environment of transmission and processing of information // Journal of Automation, Mobile Robotics and Intelligent Systems, 2018, 12(4), p. 64-69
14. Yusupbekov N.R., Abdurasulov F.R., Adilov F.T., Ivanyan A.I. Application of cloud technologies for optimization of complex processes of industrial enterprises // Advances in Intelligent Systems and Computing, 2019, 896, p. 852-858 